\title{
A LARGE DEVIATION PRINCIPLE FOR A BROWNIAN IMMIGRATION PARTICLE SYSTEM
}

\author{
MEI ZHANG, ${ }^{*}$ Beijing Normal University
}

\begin{abstract}
We derive a large deviation principle for a Brownian immigration branching particle system, where the immigration is governed by a Poisson random measure with a Lebesgue intensity measure.
\end{abstract}

Keywords: Large deviation principle; Brownian particle system; immigration process

2000 Mathematics Subject Classification: Primary 60J80

Secondary 60F05

\section{Introduction}

Consider a particle system in $\mathbb{R}^{d}$. Particles are initially distributed according to a Poisson random field with intensity measure $\mu$. Each of these particles undergoes Brownian motion until it either splits into two particles or disappears at an exponential rate. For a bounded measurable set $A \subset \mathbb{R}^{d}$, let $M_{t}(A)$ denote the number of particles in $A$ at time $t$. Define

$$
\left\langle M_{t}, f\right\rangle=\sum_{x \in \operatorname{supp} M_{t}} f(x), \quad f \in L^{1}\left(\mathbb{R}^{d}\right) .
$$

We call $\left(M_{t}\right)_{t \geq 0}$ a Brownian critical binary branching particle system; see Dawson (1993). Consider a situation in which there are additional sources of particles from which immigration occurs during the evolution. The immigration time and sites are determined by a Poisson random field on $[0, \infty) \times \mathbb{R}^{d}$ with a Lebesgue intensity measure. After arriving, each of these particles propagates and moves in $\mathbb{R}^{d}$ in the same way as the other particles. Let $N_{t}$ denote the empirical measure of the immigration particle system at $t$. The process $\left(N_{t}\right)_{t \geq 0}$ is called $a$ Brownian immigration branching particle system; see Li (1998).

The large and moderate deviation principles (LDPs and MDPs) for Brownian particle systems and super-Brownian motion have been studied by several authors; see, for example, Cox and Griffeath (1985), Deuschel and Wang (1994), Deuschel and Rosen (1998), Iscoe and Lee (1993), Lee (1993), and Hong (2003). In particular, Deuschel and Wang (1994) studied the LDP for the occupation time process of a Poisson system of independent Brownian particles without branching. The LDP for the occupation time process of branching Brownian motion was studied by Cox and Griffeath (1985). Iscoe and Lee (1993) and Lee (1993) obtained the LDPs for occupation processes of both a Brownian branching particle system and its measure-valued version. In Zhang (2004a), (2004b), the author studied the LDP and MDP for super-Brownian motion with immigration, where the speed function is $t^{1 / 2}$ for $d=1, t / \log t$ for $d=2$, and $t$ for $d \geq 3$.

Received 28 January 2005; revision received 23 May 2005.

* Postal address: School of Mathematical Science, Beijing Normal University, Beijing, 100875, P. R. China.

Email address: meizhang@bnu.edu.cn 
In this paper, we are interested in the LDP for the Brownian immigration branching particle system $\left(N_{t}\right)_{t \geq 0}$. Suppose that $N_{0}=0$, i.e. there are no particles at the initial time. For a bounded integrable function $f \geq 0$, we have

$$
\frac{1}{T}\left\langle N_{T}, f\right\rangle \rightarrow \int_{\mathbb{R}^{d}} f(x) \mathrm{d} x, \quad T \rightarrow \infty .
$$

We shall study the LDP based on this central tendency for $d=1$. In this case, the speed function is $t^{1 / 2}$, as in the case of super-Brownian motion with immigration (see Zhang (2004a)). However, in the present paper we obtain the complete LDP, while in Zhang (2004a) only a local LDP (in a neighborhood of $\int f(x) \mathrm{d} x$ ) was proved. These discussions can also be applied to super-Brownian motion with immigration, so the results of Zhang (2004a) can be modified and the complete LDP holds.

We introduce some notation before we state our results. If $u$ is a Borel measurable function on $[0, \infty) \times \mathbb{R}^{d}$, we shall often suppress a variable and write $u(t)$ for the function whose value at $x$ is $u(t, x)$. If $u$ is differentiable, we simply write $\partial u(t) / \partial t$ for $\partial u(t, x) / \partial t$, and denote by

$$
\Delta u(t) \equiv \Delta u(t, x):=\sum_{i=1}^{d} \frac{\partial^{2}}{\partial x_{i}^{2}} u(t, x)
$$

the Laplacian of $u$.

Let $C\left(\mathbb{R}^{d}\right)$ denote the space of bounded continuous functions on $\mathbb{R}^{d}$. We fix a constant $p>d$ and let $\phi_{p}(x):=\left(1+|x|^{2}\right)^{-p / 2}$ for $x \in \mathbb{R}^{d}$. Let

$$
C_{p}\left(\mathbb{R}^{d}\right):=\left\{f \in C\left(\mathbb{R}^{d}\right):|f(x)| \leq \text { const. } \times \phi_{p}(x)\right\} .
$$

The nonnegative subset of $C_{p}\left(\mathbb{R}^{d}\right)$ will be denoted by $C_{p}^{+}\left(\mathbb{R}^{d}\right)$. We denote by $M\left(\mathbb{R}^{d}\right)$ the set of all positive Radon measures on the Borel $\sigma$-algebra of $\mathbb{R}^{d}$. Let $M_{p}\left(\mathbb{R}^{d}\right) \subset M\left(\mathbb{R}^{d}\right)$ be the set of $\mu$ such that

$$
\langle\mu, f\rangle:=\int f(x) \mu(\mathrm{d} x)<\infty \text { for all } f \in C_{p}\left(\mathbb{R}^{d}\right)
$$

We endow $M_{p}\left(\mathbb{R}^{d}\right)$ with the $p$-vague topology: a sequence $\left\{\mu_{k}\right\} \subset M_{p}\left(\mathbb{R}^{d}\right)$ converges in this topology to $\mu \in M_{p}\left(\mathbb{R}^{d}\right)$ if and only if $\left\langle\mu_{k}, f\right\rangle \rightarrow\langle\mu, f\rangle$ for all $f \in C_{p}\left(\mathbb{R}^{d}\right)$. Note that the Lebesgue measure, which will always be denoted by $\lambda$, belongs to $M_{p}\left(\mathbb{R}^{d}\right)$ for $p>d$. Let $\|\cdot\|$ denote the usual supremum norm on $\mathbb{R}^{d}$. For any Lebesgue square-integrable function $\varphi$ on $[0,1] \times \mathbb{R}^{d}$, define its $L^{2}$-norm by

$$
\|\varphi(\cdot, \cdot)\|_{L^{2}\left([0,1] \times \mathbb{R}^{d}\right)}:=\left(\int_{0}^{1} \mathrm{~d} t \int_{\mathbb{R}^{d}} \varphi^{2}(t, x) \mathrm{d} x\right)^{1 / 2} .
$$

If $\left(X_{t}\right)_{t \geq 0}$ is a Markov process with state space $M_{p}\left(\mathbb{R}^{d}\right)$, we will denote by $\mathrm{P}_{\mu}$ the probability measure such that $\mathrm{P}_{\mu}\left(X_{0}=\mu\right)=1$. Expectation with respect to $\mathrm{P}_{\mu}$ will be denoted by $\mathrm{E}_{\mu}$, and $\mathrm{P}_{0}$ and $\mathrm{E}_{0}$ will be abbreviated to $\mathrm{P}$ and $\mathrm{E}$, respectively.

Suppose that $\left(P_{t}\right)_{t \geq 0}$ is the transition semigroup of Brownian motion in $\mathbb{R}^{d}$ and $p_{t}(x)$ is its density function: $p_{t}(x)=(4 \pi t)^{-d / 2} \exp \left\{-|x|^{2} / 4 t\right\}$. Suppose that $\left(M_{t}\right)_{t \geq 0}$ is the Brownian critical binary branching particle system introduced at the beginning of the paper. For $\mu \in$ 
$M_{p}\left(\mathbb{R}^{d}\right)$, let $\mathrm{E}_{(\mu)}$ denote the conditional law of $\left(M_{t}\right)_{t \geq 0}$ given that $M_{0}$ is a Poisson random measure with intensity $\mu$. Then

$$
\mathrm{E}_{(\mu)} \exp \left\{-\left\langle M_{t}, f\right\rangle\right\}=\exp \{-\langle\mu, v(t, \cdot)\rangle\}, \quad f \in C_{p}^{+}\left(\mathbb{R}^{d}\right),
$$

where $v \equiv v(t, x)$ is the unique mild solution to

$$
\frac{\partial v(t)}{\partial t}=\Delta v(t)-v^{2}(t), \quad v(0)=1-\mathrm{e}^{-f}
$$

see Dawson (1993). A Brownian immigration branching particle system $\left(N_{t}\right)_{t \geq 0}$ is an $M_{p}\left(\mathbb{R}^{d}\right)$-valued Markov process with $N_{0}=0$ and Laplace transform given by

$$
\mathrm{E} \exp \left\{-\left\langle N_{t}, f\right\rangle\right\}=\exp \left\{-\int_{0}^{t}\langle\lambda, v(s, \cdot)\rangle \mathrm{d} s\right\}, \quad f \in C_{p}^{+}\left(\mathbb{R}^{d}\right)
$$

see Li (1998).

Suppose that $f \in C_{p}^{+}\left(\mathbb{R}^{d}\right)$ and $\langle\lambda, f\rangle=1$. For $d=1$ and $T>0$, define

$$
\Lambda(T, \theta)=T^{-1 / 2} \log \mathrm{E} \exp \left\{\theta T^{-1 / 2}\left\langle N_{T}, f\right\rangle\right\} .
$$

We will prove, for some $\theta \in\left(-\infty, \theta_{0}\right), \theta_{0}>0$, that

$$
\Lambda(\theta):=\lim _{T \rightarrow \infty} \Lambda(T, \theta)=\theta+\int_{0}^{1} \mathrm{~d} s \int_{0}^{s}\left\langle\lambda,[V(r, \cdot ; \theta)]^{2}\right\rangle \mathrm{d} r,
$$

where $V(\cdot, \cdot ; \theta)$ is the unique solution to the singular PDE

$$
\frac{\partial V(s)}{\partial s}=\Delta V(s)+V^{2}(s), \quad 0 \leq s \leq 1, \quad V(0)=\theta \delta_{0} .
$$

Here $\delta_{0}$ is the Dirac mass at 0 . Moreover, $\lim _{\theta \uparrow \theta_{0}} \Lambda^{\prime}(\theta)=\infty$, where a prime denotes differentiation. Let $I(a)$ be the Legendre transform of $\Lambda(\theta)$ :

$$
I(a)=\sup _{\theta \in\left(-\infty, \theta_{0}\right)}[a \theta-\Lambda(\theta)], \quad a \in \mathbb{R} .
$$

Theorem 1.1. For $d=1$, if $U \subset \mathbb{R}$ is open and $C \subset \mathbb{R}$ is closed, then

$$
\liminf _{T \rightarrow \infty} T^{-1 / 2} \log \mathrm{P}\left\{\frac{N_{T}}{T} \in U\right\} \geq-\inf _{a \in U} I(a)
$$

and

$$
\limsup _{T \rightarrow \infty} T^{-1 / 2} \log \mathrm{P}\left\{\frac{N_{T}}{T} \in C\right\} \leq-\inf _{a \in C} I(a) .
$$

\section{Proofs}

In this section we prove Theorem 1.1. We need several supporting lemmas.

Lemma 2.1. There exists a $\theta_{0}>0$ such that, for $\theta \in\left(-\infty, \theta_{0}\right)$,

$$
\frac{\partial V(t)}{\partial t}=\Delta V(t)+V^{2}(t), \quad 0 \leq t \leq 1, \quad V(0)=\theta \delta_{0},
$$

has a unique solution $V \equiv V(t, x ; \theta)$ satisfying

$$
\lim _{\theta \uparrow \theta_{0}}\|V(\cdot, \cdot ; \theta)\|_{L^{2}([0,1] \times \mathbb{R})}=\infty .
$$


Proof. For $\theta \leq 0$, the assertation follows from Kamin and Peletier (1985, p. 205). We discuss the case $\theta>0$. In Zhang (2004a) (see Corollary 3.1 thereof), the author proved that the unique solution of $(2.1)$ exists in $L^{2}([0,1] \times \mathbb{R})$ for $\theta \in(0, \sqrt{\pi} / 4)$. Hence, it remains to determine the boundary point $\theta_{0}$. For $l>0$, consider the following equation:

$$
\frac{\partial w(s)}{\partial s}=\Delta w(s)+w^{2}(s), \quad s<l, \quad w(0)=\delta_{0} .
$$

Clearly, the solutions to (2.3) (which we denote by $\left.w \equiv w\left(s, x ; \delta_{0}\right)\right)$ and $(2.1)$ are related by

$$
w\left(s, x ; \delta_{0}\right)=\theta^{-2} V\left(\theta^{-2} s, \theta^{-1} x ; \theta\right) .
$$

Thus, (2.3) has a unique solution in $L^{2}([0, l] \times \mathbb{R})$ if $l<\pi / 16$. Define

$c=\sup \left\{t:(2.3)\right.$ has a unique solution (still denoted by $\left.w\left(\cdot, \cdot ; \delta_{0}\right)\right)$ in $\left.L^{2}([0, t] \times \mathbb{R})\right\}$.

Therefore, $c \geq \pi / 16$. In the following, we prove that $c<\infty$. Fix $t<c$ and define

$$
\bar{w}(s, x)=\int p_{t-s}(x, y) w\left(s, y ; \delta_{0}\right) \mathrm{d} y, \quad s<t .
$$

By (2.3), we have

$$
w\left(s, x ; \delta_{0}\right)=p_{s}(x)+\int_{0}^{s} P_{s-r}\left[w^{2}\left(r, \cdot ; \delta_{0}\right)\right](x) \mathrm{d} r
$$

and, so, with $p_{t}(x, y):=p_{t}(x-y)$,

$$
\begin{aligned}
\bar{w}(s, x) & =p_{t}(x)+\int p_{t-s}(x, y) \mathrm{d} y \int_{0}^{s} P_{s-r}\left[w^{2}\left(r, \cdot ; \delta_{0}\right)\right](y) \mathrm{d} r \\
& =p_{t}(x)+\int_{0}^{s} \mathrm{~d} r \int p_{t-s}(x, y) P_{s-r}\left[w^{2}\left(r, \cdot ; \delta_{0}\right)\right](y) \mathrm{d} y \\
& =p_{t}(x)+\int_{0}^{s} P_{t-r}\left[w^{2}\left(r, \cdot ; \delta_{0}\right)\right](x) \mathrm{d} r .
\end{aligned}
$$

For $s<t<c$ and $x \in \mathbb{R}$, and noting the definition of $c$, we have

$$
\begin{aligned}
\bar{w}(s, x) & \leq p_{t}(x)+\int_{0}^{s}[4 \pi(t-r)]^{-1 / 2} \mathrm{~d} r \int w^{2}\left(r, x ; \delta_{0}\right) \mathrm{d} x \\
& \leq p_{t}(x)+[4 \pi(t-s)]^{-1 / 2} \int_{0}^{s} \mathrm{~d} r \int w^{2}\left(r, x ; \delta_{0}\right) \mathrm{d} x \\
& <\infty
\end{aligned}
$$

On the other hand, by (2.4) and the Schwarz inequality, we obtain

$$
\begin{aligned}
\bar{w}(s, x) & \geq p_{t}(x)+\int_{0}^{s}\left(P_{t-r}\left[w\left(r, \cdot ; \delta_{0}\right)\right](x)\right)^{2} \mathrm{~d} r \\
& =p_{t}(x)+\int_{0}^{s} \bar{w}^{2}(r, x) \mathrm{d} r,
\end{aligned}
$$

which implies that $\bar{w}(s, x)$ is a super solution to

$$
\frac{\partial W(s, x)}{\partial s}=W^{2}(s, x), \quad s<t, \quad W(0, x)=p_{t}(x) .
$$


(That is, $\bar{w}(s, x) \geq W(s, x)$ for any $s<t$ and $x \in \mathbb{R}$.) Clearly,

$$
W(s, x)=\frac{p_{t}(x)}{1-s p_{t}(x)} .
$$

Choose $t_{0}=64 \pi$ and $x=16 \pi \sqrt{\log 2}$, meaning that $\frac{1}{2} t_{0} p_{t_{0}}(x)=1$. Let $s=\frac{1}{2} t_{0}$. Then $\bar{w}(s, x) \geq W(s, x)=\infty$, which implies that $c \leq t_{0}<\infty$. Let

$$
V(t, x ; \theta):=\theta^{2} w\left(\theta^{2} t, \theta x ; \delta_{0}\right), \quad 0<t \leq 1, x \in \mathbb{R} .
$$

Then $V(t, x ; \theta)$ is the solution of (2.1) for each $\theta<\theta_{0}:=\sqrt{c}$. To see (2.2), note that

$$
\lim _{\theta \uparrow \theta_{0}}\|V(\cdot, \cdot ; \theta)\|_{L^{2}([0,1] \times \mathbb{R})}=\theta_{0} \int_{0}^{c} \mathrm{~d} s \int w^{2}\left(s, y ; \delta_{0}\right) \mathrm{d} y .
$$

By the definition of $c$, we conclude that $\int_{0}^{c} \mathrm{~d} s \int w^{2}\left(s, y ; \delta_{0}\right) \mathrm{d} y=\infty$, and obtain (2.2).

Otherwise, if

$$
\int_{0}^{c} \mathrm{~d} s \int w^{2}\left(s, y ; \delta_{0}\right) \mathrm{d} y<\infty
$$

then let $0<\Delta c<1$. For $t \in[c, c+\Delta c]$ and $n=0,1,2, \ldots$, define

$$
\begin{aligned}
\bar{w}_{n+1}\left(t, x ; \delta_{0}\right) & =P_{t-c}\left[w\left(c, \cdot ; \delta_{0}\right)\right](x)+\int_{c}^{t} P_{t-r}\left[\bar{w}_{n}^{2}\left(r, \cdot ; \delta_{0}\right)\right](x) \mathrm{d} r \\
\bar{w}_{0}\left(t, x ; \delta_{0}\right) & =P_{t-c}\left[w\left(c, \cdot ; \delta_{0}\right)\right](x) .
\end{aligned}
$$

Define the $L^{2}$-norm of a function $g(\cdot, \cdot)$ on $[c, c+\Delta c] \times \mathbb{R}$ by

$$
\|g\|_{L^{2}}^{2}=\int_{c}^{c+\Delta c} \mathrm{~d} t \int g^{2}(t, x) \mathrm{d} x
$$

if this integral is finite. In analogy with (2.4), we have

$$
\bar{w}_{0}(t, x)=p_{t}(x)+\int_{0}^{c} P_{t-r}\left[w^{2}\left(r, \cdot ; \delta_{0}\right)\right](x) \mathrm{d} r, \quad t \in[c, c+\Delta c] .
$$

By the $C_{r}$-inequality, this implies that

$$
\begin{aligned}
\left\|\bar{w}_{0}\right\|_{L^{2}}^{2} \leq & 2 \int_{c}^{c+\Delta c} \mathrm{~d} t \int p_{t}^{2}(x) \mathrm{d} x+2 \int_{c}^{c+\Delta c} \mathrm{~d} t \int\left(\int_{0}^{c} P_{t-r}\left[w^{2}\left(r, \cdot ; \delta_{0}\right)\right](x) \mathrm{d} r\right)^{2} \mathrm{~d} x \\
= & 2 \int_{c}^{c+\Delta c} p_{2 t}(0) \mathrm{d} t+2 \int_{c}^{c+\Delta c} \mathrm{~d} t \int_{0}^{c} \mathrm{~d} r \int_{0}^{c} \mathrm{~d} u \\
& \times \iint_{2 t-r-u} p_{2}(y, z) w^{2}\left(r, y ; \delta_{0}\right) w^{2}\left(u, z ; \delta_{0}\right) \mathrm{d} y \mathrm{~d} z \\
\leq & \sqrt{\frac{2}{\pi}}(\sqrt{c+\Delta c}-\sqrt{c})+2 \int_{c}^{c+\Delta c}[4 \pi(2 t-2 c)]^{-1 / 2} \mathrm{~d} t \\
& \times \int_{0}^{c} \mathrm{~d} r \int w^{2}\left(r, y ; \delta_{0}\right) \mathrm{d} y \int_{0}^{c} \mathrm{~d} u \int w^{2}\left(u, z ; \delta_{0}\right) \mathrm{d} z .
\end{aligned}
$$


Let $A:=\int_{0}^{c} \mathrm{~d} s \int w^{2}\left(s, y ; \delta_{0}\right) \mathrm{d} y<\infty$. We then find that

$$
\left\|\bar{w}_{0}\right\|_{L^{2}}^{2} \leq \sqrt{\frac{2}{\pi}}(\sqrt{c+\Delta c}-\sqrt{c})+\sqrt{\frac{2}{\pi}} A^{2} \sqrt{\triangle c} \leq k_{1} \sqrt{\triangle c},
$$

where $k_{1}$ is a constant depending only on $c$ and $A$. In the following, we suppose that $k_{1}>1$. By the $C_{r}$-inequality and (2.6), we similarly find that

$$
\begin{aligned}
& \left\|\bar{w}_{n+1}\right\|_{L^{2}}^{2} \\
& \leq 2\left\|w_{0}\right\|_{L^{2}}^{2}+2 \int_{c}^{c+\Delta c} \mathrm{~d} t \int\left(\int_{c}^{t} P_{t-r}\left[\bar{w}_{n}^{2}\left(r, \cdot ; \delta_{0}\right)\right](x) \mathrm{d} r\right)^{2} \mathrm{~d} x \\
& \leq 2 k_{1} \sqrt{\Delta c}+2 \int_{c}^{c+\Delta c} \mathrm{~d} t \int_{c}^{t} \mathrm{~d} r \int_{c}^{t} \mathrm{~d} u \iint p_{2 t-r-u}(y, z) \bar{w}_{n}^{2}\left(r, y ; \delta_{0}\right) \bar{w}_{n}^{2}\left(u, z ; \delta_{0}\right) \mathrm{d} y \mathrm{~d} z \\
& \leq 2 k_{1} \sqrt{\Delta c}+2 \int_{c}^{c+\Delta c} \mathrm{~d} t \int_{c}^{t} \mathrm{~d} r \int_{c}^{t}[4 \pi(2 t-r-u)]^{-1 / 2} \mathrm{~d} u \\
& \quad \times 2 k_{1} \sqrt{\Delta c}+\frac{1}{\sqrt{\pi}} \int_{c}^{c+\Delta c} \mathrm{~d} t \int_{c}^{t}(t-r)^{-1 / 2} \mathrm{~d} r \int \bar{w}_{n}^{2}\left(r, y ; \delta_{0}\right) \mathrm{d} y \int_{c}^{t} \mathrm{~d} u \\
& \leq 2 k_{1} \sqrt{\Delta c}+\frac{1}{\sqrt{\pi}}\left\|\bar{w}_{n}\right\|_{L^{2}}^{2} \int_{c}^{c+\Delta c} \mathrm{~d} t \int_{c}^{t}(t-r)^{-1 / 2} \mathrm{~d} r \int \bar{w}_{n}^{2}\left(r, y ; \delta_{0}\right) \mathrm{d} y \\
& \leq 2 k_{1} \sqrt{\Delta c}+\frac{1}{\sqrt{\pi}}\left\|\bar{w}_{n}\right\|_{L^{2}}^{2} \int_{c}^{c+\Delta c} \mathrm{~d} r \int_{r}^{c+\Delta c}(t-r)^{-1 / 2} \mathrm{~d} t \int \bar{w}_{n}^{2}\left(r, y ; \delta_{0}\right) \mathrm{d} y \\
& \leq 2 k_{1} \sqrt{\Delta c}+\frac{1}{\sqrt{\pi}}\left\|\bar{w}_{n}\right\|_{L^{2}}^{2} \int_{c}^{c+\Delta c} 2 \sqrt{c+\Delta c-r} \mathrm{~d} r \int \bar{w}_{n}^{2}\left(r, y ; \delta_{0}\right) \mathrm{d} y \\
& \leq 2 k_{1} \sqrt{\Delta c}+\frac{2}{\sqrt{\pi}} \sqrt{\Delta c}\left\|_{n} \bar{w}_{n}^{4}\right\|_{L^{2}}^{4}(2.7) \\
& \leq k_{2} \sqrt{\Delta c}\left(1+\left\|\bar{w}_{n}\right\|_{L^{2}}^{4}\right), \\
& \quad \\
& \leq
\end{aligned}
$$

where $k_{2}>0$ is a constant depending only on $k_{1}$ and $c$. Choose $\Delta c, 0<\Delta c<1$, such that $\left(k_{1}+1\right) k_{2} \sqrt{\triangle c} \leq 1 \sqrt{1+\triangle c}$, and note that

$$
\left\|\bar{w}_{0}\right\|_{L^{2}}^{2} \leq k_{1} \sqrt{\triangle c} \leq k_{1} \sqrt{1+\triangle c}
$$

Suppose that $\left\|\bar{w}_{n}\right\|_{L^{2}}^{2} \leq k_{1} \sqrt{1+\triangle c}$. Recalling that we have assumed $k_{1}>1$, by (2.7) we have

$$
\begin{aligned}
\left\|\bar{w}_{n+1}\right\|_{L^{2}}^{2} & \leq k_{2} \sqrt{\triangle c}\left[1+k_{1}^{2}(1+\Delta c)\right] \\
& \leq k_{2}\left(k_{1}^{2}+1\right) \sqrt{\triangle c}(1+\Delta c) \\
& \leq k_{2}\left(k_{1}^{2}+k_{1}\right) \sqrt{\triangle c}(1+\triangle c) \\
& \leq k_{1} \sqrt{1+\Delta c} \\
& <\infty
\end{aligned}
$$


From (2.6), we know that $\bar{w}_{n}$ is increasing in $n$. Therefore, $\bar{w}:=\lim _{n \rightarrow \infty} \bar{w}_{n}$ exists in $L^{2}([c, c+\Delta c] \times \mathbb{R})$ and satisfies $(2.3)$ on the interval $[c, c+\triangle c]$.

It is easy to check that

$$
\tilde{w}\left(t, x ; \delta_{0}\right)= \begin{cases}w\left(t, x ; \delta_{0}\right), & t \in[0, c], \\ \bar{w}\left(t, x ; \delta_{0}\right), & t \in[c, c+\Delta c],\end{cases}
$$

is a solution to (2.3) that remains in $L^{2}([c, c+\Delta c] \times \mathbb{R})$. Let us see the uniqueness of this solution. Suppose that $\tilde{w}_{1}$ is another solution to $(2.3)$ in $L^{2}([c, c+\Delta c] \times \mathbb{R})$. Then

$$
\begin{array}{cll}
\tilde{w}\left(t, x ; \delta_{0}\right)=P_{t-c}\left[w\left(c, \cdot ; \delta_{0}\right)\right](x)+\int_{c}^{t} P_{t-r}\left[\tilde{w}^{2}\left(r, \cdot ; \delta_{0}\right)\right](x) \mathrm{d} r, & t \in[c, c+\Delta c], \\
\tilde{w}_{1}\left(t, x ; \delta_{0}\right)=P_{t-c}\left[w\left(c, \cdot ; \delta_{0}\right)\right](x)+\int_{c}^{t} P_{t-r}\left[\tilde{w}_{1}^{2}\left(r, \cdot ; \delta_{0}\right)\right](x) \mathrm{d} r, & t \in[c, c+\Delta c] .
\end{array}
$$

By using the Schwarz inequality and arguing as in (2.7), we find that

$$
\begin{aligned}
J & :=\int_{c}^{c+\Delta c} \mathrm{~d} t \int\left|\tilde{w}\left(t, x ; \delta_{0}\right)-\tilde{w}_{1}\left(t, x ; \delta_{0}\right)\right|^{2} \mathrm{~d} t \mathrm{~d} x \\
& =\int_{c}^{c+\Delta c} \mathrm{~d} t \int\left(\int_{c}^{t} P_{t-r}\left[\left|\tilde{w}^{2}\left(r, \cdot ; \delta_{0}\right)-\tilde{w}_{1}^{2}\left(r, \cdot ; \delta_{0}\right)\right|\right](x) \mathrm{d} r\right)^{2} \mathrm{~d} x \\
& \leq \frac{2}{\sqrt{\pi}}\|\tilde{w}\|_{L^{2}}^{2}\left\|\tilde{w}_{1}\right\|_{L^{2}}^{2} \int_{c}^{c+\Delta c} \sqrt{c+\Delta c-r} \mathrm{~d} r \int\left|\tilde{w}\left(r, y ; \delta_{0}\right)-\tilde{w}_{1}\left(r, y ; \delta_{0}\right)\right|^{2} \mathrm{~d} y \\
& \leq \frac{2}{\sqrt{\pi}}\|\tilde{w}\|_{L^{2}}^{2}\left\|\tilde{w}_{1}\right\|_{L^{2}}^{2} \sqrt{\Delta c} J .
\end{aligned}
$$

Since both $\tilde{w}$ and $\tilde{w}_{1}$ belong to $L^{2}([c, c+\Delta c] \times \mathbb{R})$, we can choose $\Delta c>0$ such that

$$
\frac{2}{\sqrt{\pi}}\|\tilde{w}\|_{L^{2}}^{2}\left\|\tilde{w}_{1}\right\|_{L^{2}}^{2} \sqrt{\triangle c}<1 .
$$

Then we have

$$
J=\int_{c}^{c+\Delta c} \mathrm{~d} t \int\left|\tilde{w}\left(t, x ; \delta_{0}\right)-\tilde{w}_{1}\left(t, x ; \delta_{0}\right)\right|^{2} \mathrm{~d} x=0 .
$$

Therefore, if

$$
\int_{0}^{c} \mathrm{~d} s \int w^{2}\left(s, y ; \delta_{0}\right) \mathrm{d} y<\infty
$$

then by (2.8) we can construct the unique solution of (2.3) in $L^{2}([0, c+\Delta c] \times \mathbb{R})$. This contradicts the definition of $c$, and the proof is complete.

Corollary 2.1. For each $\theta \in\left(-\infty, \theta_{0}\right)$, there exists an $a>1$ such that

$$
\frac{\partial U(t)}{\partial t}=\Delta U(t)+a U^{2}(t), \quad 0<t \leq 1, \quad U(0)=\theta \delta_{0},
$$

has a unique solution $U(\cdot, \cdot ; \theta) \in L^{2}([0,1] \times \mathbb{R})$. 
Proof. Set $\tilde{U}=a U$, where $U$ is the solution to (2.9). Then $\tilde{U}$ is the solution to

$$
\frac{\partial \tilde{U}(t)}{\partial t}=\Delta \tilde{U}(t)+\tilde{U}^{2}(t), \quad 0<t \leq 1, \quad \tilde{U}(0)=a \theta \delta_{0} .
$$

For each $\theta \in\left(-\infty, \theta_{0}\right)$, we can choose an $a>1$ such that $a \theta \in\left(-\infty, \theta_{0}\right)$. By Lemma 2.1, (2.10) has a unique solution $\tilde{U}$, and the assertion follows.

Lemma 2.2. Suppose that $f \in C_{p}^{+}\left(\mathbb{R}^{d}\right)$ with $\langle\lambda, f\rangle=1$. Let

$$
f_{T}(x, \theta)=T\left(\exp \left\{\theta T^{-1 / 2} f\left(T^{1 / 2} x\right)\right\}-1\right) .
$$

For $\theta \in\left(-\infty, \theta_{0}\right)$, the equation

$$
\frac{\partial V_{T}(t)}{\partial t}=\Delta V_{T}(t)+V_{T}^{2}(t), \quad 0 \leq t \leq 1, \quad V_{T}(0)=f_{T}(\cdot, \theta),
$$

has a unique solution $V_{T}(\cdot, \cdot ; \theta)$, which converges to $V(\cdot, \cdot ; \theta)$ in $L^{2}([0,1] \times \mathbb{R})$ as $T \rightarrow \infty$. If we allow $\theta$ to be a complex number such that $|\theta|<\theta_{0}$, then $V_{T}(\cdot, \cdot ; \theta)$ is analytic in $|\theta|<\theta_{0}$.

To prove Lemma 2.2 we need the following result.

Lemma 2.3. As $T \rightarrow \infty$,

$$
\int_{0}^{1} \mathrm{~d} t \int\left(P_{t}\left[f_{T}(\cdot, \theta)\right](x)-\theta p_{t}(x)\right)^{2} \mathrm{~d} x \rightarrow 0 .
$$

Proof. If $\theta=0$ the assertion is obvious. For $\theta \neq 0$,

$$
\begin{aligned}
\int_{0}^{1} \mathrm{~d} t \int\left(P_{t}\left[f_{T}(\cdot, \theta)\right](x)-\theta p_{t}(x)\right)^{2} \mathrm{~d} x= & \int_{0}^{1} \mathrm{~d} t \iint p_{2 t}(x, y) f_{T}(x, \theta) f_{T}(y, \theta) \mathrm{d} x \mathrm{~d} y \\
& -2 \theta \int_{0}^{1} \mathrm{~d} t \int p_{2 t}(x, 0) f_{T}(x, \theta) \mathrm{d} x \\
& +\theta^{2} \int_{0}^{1} p_{2 t}(0) \mathrm{d} t
\end{aligned}
$$

For the first term on the right-hand side of (2.12), we have

$$
\begin{aligned}
I_{1} & :=\int_{0}^{1} \mathrm{~d} t \iint p_{2 t}(x, y) f_{T}(x, \theta) f_{T}(y, \theta) \mathrm{d} x \mathrm{~d} y \\
& =\int_{0}^{1} \mathrm{~d} t \iint p_{2 t}\left(T^{-1 / 2}(w-u)\right) T\left(\mathrm{e}^{\theta T^{-1 / 2} f(w)}-1\right)\left(\mathrm{e}^{\theta T^{-1 / 2} f(u)}-1\right) \mathrm{d} w \mathrm{~d} u .
\end{aligned}
$$

After simple calculation, we find that

$$
\left|\mathrm{e}^{x}-1\right|< \begin{cases}a|x|, & 0<x<\log a, a>1 \\ |x|, & x \leq 0\end{cases}
$$

For $T>T_{0}(\theta, f):=(\theta f)^{2}(\log 2)^{-2}$ we have

$$
\left|\theta T^{-1 / 2} f(x)\right| \leq|\theta|\|f\| T^{-1 / 2}<\log 2 .
$$


Then

$$
T^{1 / 2}\left|\left(\mathrm{e}^{\theta T^{-1 / 2} f(w)}-1\right)\right| \leq 2|\theta f(w)| .
$$

By applying the dominated convergence theorem to $I_{1}$, and recalling that $\langle\lambda, f\rangle=1$, we find that

$$
I_{1} \rightarrow \theta^{2} \int_{0}^{1} p_{2 t}(0) \mathrm{d} t<\infty
$$

Similarly, we obtain

$$
I_{2}:=2 \theta \int_{0}^{1} \mathrm{~d} t \int p_{2 t}(x, 0) f_{T}(x, \theta) \mathrm{d} x \rightarrow 2 \theta^{2} \int_{0}^{1} p_{2 t}(0) \mathrm{d} t .
$$

This completes the proof.

Proof of Lemma 2.2. For $\theta \leq 0$, by replacing $f$ by $-\theta T^{-1 / 2} f\left(T^{1 / 2}\right.$.) in (1.2) and making the change of variable $V(t) \leftrightarrow-V(t)$, we obtain (2.11). For $0<\theta<\theta_{0}$, define

$$
\bar{V}_{T}(s, x, \theta)=\int \frac{1}{\theta} f_{T}(x-y, \theta) U(s, y ; \theta) \mathrm{d} y,
$$

where $U(\cdot, \cdot ; \theta)$ is the solution to (2.9). By (2.1) and the Schwarz inequality, we have

$$
\begin{aligned}
& \bar{V}_{T}(s, x, \theta) \\
& \quad=P_{s}\left[f_{T}(\cdot, \theta)\right](x)+a \int_{0}^{s} \mathrm{~d} r \int \frac{1}{\theta} f_{T}(x-y, \theta) P_{s-r}\left[U^{2}(r, \cdot ; \theta)\right](y) \mathrm{d} y \\
& =P_{s}\left[f_{T}(\cdot, \theta)\right](x)+\frac{a}{\theta} \int_{0}^{s} \mathrm{~d} r \iint f_{T}(x-y, \theta) p_{s-r}(y, z) U^{2}(r, z ; \theta) \mathrm{d} y \mathrm{~d} z \\
& =P_{s}\left[f_{T}(\cdot, \theta)\right](x)+\frac{a}{\theta} \int_{0}^{s} \mathrm{~d} r \iint p_{s-r}(w) f_{T}(x-w-z, \theta) U^{2}(r, z ; \theta) \mathrm{d} w \mathrm{~d} z \\
& \geq P_{s}\left[f_{T}(\cdot, \theta)\right](x)+\frac{a}{\theta} \int_{0}^{s} \mathrm{~d} r \int p_{s-r}(w) \mathrm{d} w\left(\int f_{T}(x-w-z, \theta) U(r, z ; \theta) \mathrm{d} z\right)^{2} \\
& \quad \geq P_{s}\left[f_{T}(\cdot, \theta)\right](x)+\frac{1}{\theta^{2}} \int_{0}^{s} \mathrm{~d} r \int p_{s-r}(w) \mathrm{d} w\left(\int f_{T}(x-w-z, \theta) U(r, z ; \theta) \mathrm{d} z\right)^{2} \\
& =P_{s}\left[f_{T}(\cdot, \theta)\right](x)+\int_{0}^{s} \mathrm{~d} r \int P_{s-r}\left[\bar{V}_{T}^{2}(r, \cdot, \theta)\right] \mathrm{d} r .
\end{aligned}
$$

In the fifth step, we have used (2.13), which yields

$$
\begin{aligned}
\left(\int f_{T}(x-w-z, \theta) \mathrm{d} z\right)^{-1} & =\left(T \int\left(\exp \left\{\theta T^{-1 / 2} f\left(T^{1 / 2}(x-w-z)\right)\right\}-1\right) \mathrm{d} z\right)^{-1} \\
& \geq\left(a \theta T^{1 / 2} \int f\left(T^{1 / 2}(x-w-z)\right) \mathrm{d} z\right)^{-1} \\
& =(a \theta)^{-1}
\end{aligned}
$$

when $T$ is sufficiently large. 
Thus, $\bar{V}_{T}$ is a super solution of (2.11), i.e.

$$
\bar{V}_{T}(s, x ; \theta) \geq V_{T}(s, x ; \theta)
$$

for any $(s, x) \in[0,1] \times \mathbb{R}$. By standard results on differential equations, we conclude that (2.11) has a unique solution $V_{T}$ for $\theta \in\left(0, \theta_{0}\right)$.

In the following we discuss the limit of $V_{T}$. By (2.11) and (2.1) we have, for $0<\varepsilon<1$,

$$
\begin{aligned}
I(T):= & \int_{0}^{\varepsilon} \mathrm{d} t \int\left|V_{T}(t, x ; \theta)-V(t, x ; \theta)\right|^{2} \mathrm{~d} x \\
\leq & 2 \int_{0}^{\varepsilon} \mathrm{d} t \int\left(P_{t}\left[f_{T}(x, \theta)\right]-\theta p_{t}(x)\right)^{2} \mathrm{~d} x \\
& +2 \int_{0}^{\varepsilon} \mathrm{d} t \int\left(\int_{0}^{t} P_{t-r}\left[\left|V_{T}^{2}(r)-V^{2}(r)\right|\right](x) \mathrm{d} r\right)^{2} \mathrm{~d} x \\
= & : 2 I_{1}(T)+2 I_{2}(T),
\end{aligned}
$$

where

$$
I_{1}(T)=\int_{0}^{\varepsilon} \mathrm{d} t \int\left(P_{t}\left[f_{T}(x, \theta)\right]-\theta p_{t}(x)\right)^{2} \mathrm{~d} x \rightarrow 0
$$

according to Lemma 2.3. By the Schwarz inequality, in analogy with (2.7) we have

$$
\begin{aligned}
& I_{2}(T)= \int_{0}^{\varepsilon} \mathrm{d} t \int\left(\int_{0}^{t} P_{t-r}\left[\left|V_{T}^{2}(r)-V^{2}(r)\right|\right](x) \mathrm{d} r\right)^{2} \mathrm{~d} x \\
&= \int_{0}^{\varepsilon} \mathrm{d} t \int_{0}^{t} \mathrm{~d} s \int_{0}^{t} \mathrm{~d} r \iint p_{2 t-s-r}(x, y)\left|V_{T}(r)-V(r)\right|^{2}(x) \\
& \times\left|V_{T}(s)+V(s)\right|^{2}(y) \mathrm{d} x \mathrm{~d} y \\
& \leq(4 \pi)^{-1 / 2} \int_{0}^{\varepsilon} \mathrm{d} t \int_{0}^{t} \mathrm{~d} r \int\left|V_{T}(r, x)-V(r, x)\right|^{2} \mathrm{~d} x \\
& \leq \times \int_{0}^{t}(2 t-s-r)^{-1 / 2} \mathrm{~d} s \int\left(V_{T}^{2}(s, y)+V^{2}(s, y)\right) \mathrm{d} y \\
& \leq \int_{0}^{\varepsilon} \mathrm{d} t \int_{0}^{t}(t-r)^{-1 / 2} \mathrm{~d} r \int\left|V_{T}(r, x)-V(r, x)\right|^{2} \mathrm{~d} x \\
& \times \int_{0}^{t} \mathrm{~d} s \int\left(\bar{V}_{T}^{2}(s, y)+V^{2}(s, y)\right) \mathrm{d} y .
\end{aligned}
$$

For any $x \in \mathbb{R}$, using (2.13) we obtain

$$
\frac{1}{\theta} \int\left|f_{T}(x-y, \theta)\right| \mathrm{d} y=\frac{1}{\theta} \int\left|f_{T}(x-y, \theta)\right| \mathrm{d} x=\frac{1}{\theta} \int\left|f_{T}(z, \theta)\right| \mathrm{d} z \leq M(\theta, f)
$$


for $T>T_{0}$, where $M(\theta, f)>0$ is a constant depending only on $\theta$ and $f$. Then, by the Schwarz inequality, we have

$$
\begin{aligned}
& \int_{0}^{1} \int \bar{V}_{T}^{2}(s, x) \mathrm{d} x \mathrm{~d} s \\
& \quad=\int_{0}^{1} \mathrm{~d} s \int\left(\frac{1}{\theta} \int f_{T}(x-y, \theta) U(s, y ; \theta) \mathrm{d} y\right)^{2} \mathrm{~d} x \\
& \quad \leq \frac{1}{\theta^{2}} \int_{0}^{1} \mathrm{~d} s \int \mathrm{d} x\left(\int\left|f_{T}(x-y, \theta)\right| U^{2}(s, y ; \theta) \mathrm{d} y\right)\left(\int\left|f_{T}(x-y)\right| \mathrm{d} y\right) \\
& \quad \leq \frac{M(\theta, f)}{\theta} \int_{0}^{1} \mathrm{~d} s \int U^{2}(s, y ; \theta) \mathrm{d} y \int\left|f_{T}(x-y, \theta)\right| \mathrm{d} x \\
& \quad \leq M^{2}(\theta, f) \int_{0}^{1} \mathrm{~d} s \int U^{2}(s, y ; \theta) \mathrm{d} y \\
& \quad<\infty .
\end{aligned}
$$

By Lemma 2.1, we have

$$
\int_{0}^{1} \mathrm{~d} s \int V^{2}(s, y ; \theta) \mathrm{d} y<\infty
$$

By combining these results, for $0<\varepsilon<1$ we find that

$$
\begin{aligned}
I_{2}(T) & \leq C \int_{0}^{\varepsilon} \mathrm{d} t \int_{0}^{t}(t-r)^{-1 / 2} \mathrm{~d} r \int\left|V_{T}(r, x)-V(r, x)\right|^{2} \mathrm{~d} x \\
& \leq C \int_{0}^{\varepsilon} \mathrm{d} r \int_{r}^{\varepsilon}(t-r)^{-1 / 2} \mathrm{~d} t \int\left|V_{T}(r, x)-V(r, x)\right|^{2} \mathrm{~d} x \\
& \leq C \int_{0}^{\varepsilon} 2(\varepsilon-r)^{1 / 2} \mathrm{~d} r \int\left|V_{T}(r, x)-V(r, x)\right|^{2} \mathrm{~d} x \\
& \leq 2 C \varepsilon^{1 / 2} \int_{0}^{\varepsilon} \mathrm{d} r \int\left|V_{T}(r, x)-V(r, x)\right|^{2} \mathrm{~d} x \\
& =2 C \varepsilon^{1 / 2} I(T),
\end{aligned}
$$

where $C>0$ is a constant. Recalling (2.14), we have

$$
I(T) \leq 2 I_{1}(T)+4 C \varepsilon^{1 / 2} I(T) .
$$

By choosing an $\varepsilon>0$ such that $4 C \varepsilon^{1 / 2}<1$, we see that

$$
I(T) \leq 2\left(1-4 C \varepsilon^{1 / 2}\right)^{-1} I_{1}(T) \rightarrow 0
$$

as $T \rightarrow \infty$. Now consider dividing $[0,1]$ into intervals $[0, \varepsilon],[\varepsilon, 2 \varepsilon], \ldots$ By similar methods we can prove that

$$
\int_{\varepsilon}^{2 \varepsilon} \mathrm{d} r \int\left(V_{T}(r, x)-V(r, x)\right)^{2} \mathrm{~d} x \rightarrow 0, \quad n=0,1,2, \ldots
$$

In fact, for $t \in[\varepsilon, 2 \varepsilon]$ we have

$$
\begin{gathered}
V_{T}(t, x ; \theta)=P_{t-\varepsilon}\left[V_{T}(\varepsilon, \cdot ; \theta)\right](x)+\int_{\varepsilon}^{t} P_{t-r}\left[V_{T}^{2}(r, \cdot ; \theta)\right] \mathrm{d} r, \\
V(t, x ; \theta)=P_{t-\varepsilon}[V(\varepsilon, \cdot ; \theta)](x)+\int_{\varepsilon}^{t} P_{t-r}\left[V^{2}(r, \cdot ; \theta)\right] \mathrm{d} r .
\end{gathered}
$$


In analogy with (2.14), define

$$
I^{\prime}(T)=\int_{\varepsilon}^{2 \varepsilon} \mathrm{d} t \int\left|V_{T}(t, x ; \theta)-V(t, x ; \theta)\right|^{2} \mathrm{~d} x .
$$

As $T \rightarrow \infty$,

$$
\begin{aligned}
I_{1}^{\prime}(T) & :=\int_{\varepsilon}^{2 \varepsilon} \mathrm{d} t \int\left(P_{t-\varepsilon}\left[V_{T}(\varepsilon, \cdot ; \theta)\right](x)-P_{t-\varepsilon}[V(\varepsilon, \cdot ; \theta)](x)\right)^{2} \mathrm{~d} x \\
& \leq \int_{\varepsilon}^{2 \varepsilon} \mathrm{d} t \int\left(P_{\varepsilon}\left[V_{T}(t-\varepsilon, \cdot ; \theta)(x)-V(t-\varepsilon, \cdot ; \theta)\right](x)\right)^{2} \mathrm{~d} x \\
& =\int_{\varepsilon}^{2 \varepsilon} \mathrm{d} t \int\left(V_{T}(t-\varepsilon, \cdot ; \theta)(x)-V(t-\varepsilon, \cdot ; \theta)(x)\right)^{2} \mathrm{~d} x \\
& =I_{1}(T) \\
& \rightarrow 0
\end{aligned}
$$

The remainder of the proof of (2.15) is analogous to our previous discussions on $I(T)$. Clearly there are no more than $\lfloor 1 / \varepsilon\rfloor+1$ such intervals of the form $[n \varepsilon,(n+1) \varepsilon]$, where $\lfloor\cdot\rfloor$ is the least-integer function. Therefore we arrive at

$$
\int_{0}^{1} \mathrm{~d} r \int\left(V_{T}(r, x)-V(r, x)\right)^{2} \mathrm{~d} x \rightarrow 0 .
$$

Now let us see the analyticity of $V_{T}(t, x ; \theta)$. For each $T>0$, define $\left\{v_{T}^{(n)}, n \in \mathbb{N}\right\}$ recursively by

$$
\begin{aligned}
v_{T}^{(0)}(t, x ; \theta) & =0, \\
v_{T}^{(n+1)}(t, x ; \theta) & =P_{t}\left[f_{T}(\cdot, \theta)\right](x)+\int_{0}^{t} P_{t-s}\left[v_{T}^{(n)}(s, \cdot ; \theta)\right]^{2}(x) \mathrm{d} s .
\end{aligned}
$$

By induction, for each real number $\theta \in\left(0, \theta_{0}\right)$, we obtain $0 \leq v_{T}^{(n)}(t, x ; \theta) \leq \bar{V}_{T}(t, x ; \theta)$ for all $n, t$, and $x$, and $v_{T}^{(n)}$ is increasing in $n$. By applying the monotone convergence theorem to (2.16), for each $\theta \in\left(0, \theta_{0}\right)$ we find that

$$
v_{T}^{(n)}(t, x ; \theta) \rightarrow V_{T}(t, x ; \theta) \quad \text { as } n \rightarrow \infty .
$$

Now allow $\theta$ to be a complex number. Again by induction, it is easy to see that, for $n \in N$, $v_{T}^{(n)}(t, x ; \theta)$ is analytic in $|\theta|<\theta_{0}$ and

$$
\sup _{n}\left|v_{T}^{(n)}(t, x ; \theta)\right| \leq V_{T}(t, x ;|\theta|), \quad|\theta|<\theta_{0} .
$$

Thus, $\left\{v_{T}^{(n)}(t, x ; \theta), n \in \mathbb{N}\right\}$ is a normal family of analytic functions on the disc $|\theta|<\theta_{0}$, and $v_{T}^{(n)}(t, x ; \theta) \rightarrow V_{T}(t, x ; \theta)$ for $\theta$ in the real interval $\left(0, \theta_{0}\right)$. Therefore, by Conway (1978, p. 151, Theorem 2.1) and Vitali's theorem (see Conway (1978, p. 154)), the sequence

$$
\left\{v_{T}^{(n)}(t, x ; \theta), n \in \mathbb{N}\right\}
$$

converges to an analytic function $V_{T}(t, x ; \theta)$ on the disc $|\theta|<\theta_{0}$. 
Corollary 2.2. Suppose that $\Lambda(\theta)$ is defined by (1.4). As $T \rightarrow \infty$, we have

$$
\Lambda(\theta)=\theta+\int_{0}^{1} \mathrm{~d} s \int_{0}^{s}\left\langle\lambda,[V(r, \cdot ; \theta)]^{2}\right\rangle \mathrm{d} r
$$

and $\lim _{\theta \uparrow \theta_{0}} \Lambda^{\prime}(\theta)=\infty$.

Proof. For $\theta \leq 0$, by (1.3) and (1.1) we have

$$
\Lambda(T, \theta)=T^{-1 / 2} \log \operatorname{Eexp}\left\{\left\langle N_{T}, \theta T^{-1 / 2} f\right\rangle\right\}=T^{-1 / 2} \int_{0}^{T} \mathrm{~d} r \int \tilde{v}\left(r, x ; \theta T^{-1 / 2}\right) \mathrm{d} x
$$

where $\tilde{v}\left(\cdot, \cdot ; \theta T^{-1 / 2}\right)$ is the solution to

$$
\frac{\partial \tilde{v}(t)}{\partial t}=\Delta \tilde{v}(t)+\tilde{v}^{2}(t), \quad 0<t \leq 1, \quad \tilde{v}(0)=\mathrm{e}^{\theta T^{-1 / 2} f}-1
$$

By changing variables according to $r=u T$ and $x=T^{1 / 2} y$, we obtain

$$
\Lambda(T, \theta)=\int_{0}^{1}\left\langle\lambda, V_{T}(u, \cdot ; \theta)\right\rangle \mathrm{d} u=\left\langle\lambda, f_{T}(\cdot, \theta)\right\rangle+\int_{0}^{1} \mathrm{~d} s \int_{0}^{s}\left\langle\lambda,\left[V_{T}(r, \cdot ; \theta)\right]^{2}\right\rangle \mathrm{d} r
$$

for $\theta \leq 0$, where $f_{T}$ is as given in Lemma 2.2 and $V_{T}(\cdot, \cdot ; \theta)$ is the solution to (2.11). Now allow $\bar{\theta}$ to be a complex variable. For $|\theta|<\theta_{0}$, the analyticity of $f_{T}(x, \theta)$ and $V_{T}(t, x ; \theta)$ implies that

$$
\Gamma(T, \theta):=\left\langle\lambda, f_{T}(\cdot, \theta)\right\rangle+\int_{0}^{1} \mathrm{~d} s \int_{0}^{s}\left\langle\lambda,\left[V_{T}(r, \cdot ; \theta)\right]^{2}\right\rangle \mathrm{d} r
$$

is an analytic function on the disc $|\theta|<\theta_{0}$. For a fixed $f$ and $T$, denote the law of $\left\langle N_{T}\right.$, $\left.T^{-1 / 2} f\right\rangle$ by $\mu_{T}$. Then $\operatorname{Eexp}\left\{\left\langle N_{T}, \theta T^{-1 / 2} f\right\rangle\right\}$ is the Laplace transform of the probability law $\mu_{T}$ on $[0, \infty)$. By Widder (1941, p. 57, Theorem 5a), $\operatorname{Eexp}\left\{\left\langle N_{T}, \theta T^{-1 / 2} f\right\rangle\right\}$ and, thus, $\Lambda(T, \theta)$ are analytic in the half-plane $\{\theta: \theta=\sigma+i \tau, \sigma<0\}$. For each real number $\theta<0$, we then have

$$
\Lambda(T, \theta)=\Gamma(T, \theta)
$$

Therefore, by the uniqueness of analytic extension, $\Lambda(T, \theta)$ can be uniquely extended to the real line-segment $\left[0, \theta_{0}\right)$, upon which it coincides with $\Gamma(T, \theta)$. Thus, $(2.18)$ holds for the real number $\theta,-\infty<\theta<\theta_{0}$. Let $T \rightarrow \infty$. Then, noting the two formulae below (2.13), we have $\left\langle\lambda, f_{T}(\cdot, \theta)\right\rangle \rightarrow \theta$, and by applying Lemma 2.2 we recover $(2.17)$. By $(2.5)$, for $\theta \in\left(0, \theta_{0}\right)$ we have

$$
\begin{aligned}
\Lambda(\theta) & =\theta+\theta^{4} \int_{0}^{1} \mathrm{~d} s \int_{0}^{s} \mathrm{~d} r \int w^{2}\left(\theta^{2} r, \theta x ; \delta_{0}\right) \mathrm{d} x \\
& =\theta+\theta^{-1} \int_{0}^{\theta^{2}} \mathrm{~d} s \int_{0}^{s} \mathrm{~d} r \int w^{2}\left(r, y ; \delta_{0}\right) \mathrm{d} y,
\end{aligned}
$$


and, so,

$$
\begin{aligned}
\Lambda^{\prime}(\theta) & =1-\theta^{-2} \int_{0}^{\theta^{2}} \mathrm{~d} s \int_{0}^{s} \mathrm{~d} r \int w^{2}\left(r, y ; \delta_{0}\right) \mathrm{d} y+2 \int_{0}^{\theta^{2}} \mathrm{~d} r \int w^{2}\left(r, y ; \delta_{0}\right) \mathrm{d} y \\
& \geq 1-\theta^{-2} \int_{0}^{\theta^{2}} \mathrm{~d} s \int_{0}^{\theta^{2}} \mathrm{~d} r \int w^{2}\left(r, y ; \delta_{0}\right) \mathrm{d} y+2 \int_{0}^{\theta^{2}} \mathrm{~d} r \int w^{2}\left(r, y ; \delta_{0}\right) \mathrm{d} y \\
& =1+\int_{0}^{\theta^{2}} \mathrm{~d} r \int w^{2}\left(r, y ; \delta_{0}\right) \mathrm{d} y \\
& =1+\|V(\cdot, ; \theta)\|_{L^{2}([0,1] \times \mathbb{R})}^{2} .
\end{aligned}
$$

Thus, the second assertion follows from Lemma 2.1, and the proof is complete.

Proof of Theorem 1.1. This is immediate from Corollary 2.2 and the Gärtner-Ellis theorem (see Dembo and Zeitouni (1998)).

\section{Acknowledgement}

I would like to thank the referee for his valuable comments and suggestions.

\section{References}

Conway, J. B. (1978). Functions of One Complex Variable. Springer, New York.

Cox, J. T. and Griffeath, D. (1985). Occupation times for critical branching Brownian motions. Ann. Prob. 13, 1108-1132.

Dawson, D. A. (1993). Measure-valued Markov processes. In École d'Été de Probabilités de Saint-Flour XXI (Lecture Notes. Math. 1541), Springer, Berlin, pp. 1-260.

Dembo, A. And Zeitouni, O. (1998). Large Deviations Techniques and Applications. Springer, New York.

Deuschel, J. D. And Rosen, J. (1998). Occupation time large deviations for critical branching Brownian motion, super-Brownian motion and related processes. Ann. Prob. 26, 602-643.

Deuschel, J. D. AND Wang, K. M. (1994). Large deviations for the occupation time functional of a Poisson system of independent Brownian particles. Stoch. Process. Appl. 52, 183-209.

Hong, W. M. (2003). Large deviations for the super-Brownian motion with super-Brownian immigration. J. Theoret. Prob. 16, 899-922.

IsCOE, I. AND LEE, T. Y. (1993). Large deviations for occupation times of measure-valued branching Brownian motions. Stoch. Stoch. Reports 45, 177-209.

Kamin, S. and Peletier, L. A. (1985). Singular solutions of the heat equation with absorption. Proc. Amer. Math. Soc. 95, 205-210.

LeE, T. Y. (1993). Some limit theorems for super-Brownian motion and semilinear differential equations. Ann. Prob. 21, 979-995.

LI, Z. H. (1998). Immigration processes associated with branching particle systems. Adv. Appl. Prob. 30, 657-675.

WidDER, D. V. (1941). The Laplace Transform. Princeton University Press.

Zhang, M. (2004a). Large deviations for super-Brownian motion with immigration. J. Appl. Prob. 41, 187-201.

Zhang, M. (2004b). Moderate deviation for super-Brownian motion with immigration. Sci. China Ser. A 47, 440-452. 